\title{
A 'serious' bloodstream infection in an infant
}

\author{
Shauna Flavelle $\mathrm{BSc}^{1,2}$, Gregory J Tyrrell $\mathrm{PhD}^{3}$, Sarah E Forgie $\mathrm{MD}^{1,2}$
}

\begin{abstract}
A 10-month-old boy was brought to the emergency department with a four-day history of an erythematous central line insertion site, five months following a liver-small bowel-pancreas transplant. One day before presentation, there had been some purulent discharge from the site. Other than increased fussiness, there was no documented fever, vomiting, change in appetite or change in bowel habits. The child had not had any recent infections and no known sick contacts. He went swimming for the first time two weeks before admission, but otherwise he had no new exposures. His travel history was unremarkable. The child ate table foods, and a family favourite was Chinese food, which they ate several times a week.

The patient was born at 35 weeks' gestation with gastroschisis. Within three weeks, he underwent a staged repair and after failing a refeeding protocol, he was started on total parenteral nutrition. At five months of age, he underwent a liversmall bowel-pancreas transplant for complications related to his gastroschisis, including total parenteral nutrition cholestasis, and he was sent home at seven months of age. His central venous line had been in place since his transplant. Current line care involved flushing three times a week by home care nurses, and regular cleaning with alcohol swabs by his parents.
\end{abstract}

Medications included tacrolimus, sirolimus, prednisone, trimethoprim-sulfamethoxazole, ranitidine and ferrous sulphate. His immunizations were up to date and his family history was unremarkable.

Physical examination revealed a nontoxic-looking 10 -month-old in no apparent distress. His temperature was $36.9^{\circ} \mathrm{C}$, pulse rate was 139 beats $/ \mathrm{min}$ and respiratory rate was 36 breaths/min. A small margin around the catheter site appeared erythematous with a small amount of purulent exudate. The area around the line insertion site was not tender to palpation. Aside from surgical scars, the remainder of his examination was unremarkable.

Hematological studies revealed a white blood cell count of $4.8 \times 10^{9} / \mathrm{L}$ with $1.7 \times 10^{9} / \mathrm{L}$ neutrophils and $2.1 \times 10^{9} / \mathrm{L}$ lymphocytes. Hemoglobin level was $106 \mathrm{~g} / \mathrm{L}$ and platelet count was $254 \times 10^{9} / \mathrm{L}$. Electrolyte, creatinine and urea levels were all unremarkable. Alkaline phosphatase was elevated, at $389 \mathrm{U} / \mathrm{L}$, while alanine aminotransferase, aspartate aminotransferase and total bilirubin levels were all normal. A swab performed at the line insertion site grew coagulase-negative Staphylococcus.

What did the blood culture reveal?

\footnotetext{
${ }^{1}$ Stollery Children's Hospital; ${ }^{2}$ Department of Pediatrics; ${ }^{3}$ Department of Laboratory Medicine and Pathology, University of Alberta, Edmonton, Alberta

Correspondence: Dr Sarah E Forgie, Stollery Children's Hospital, Department of Pediatrics, Room 8213, Aberhart Centre One, 11402 University Avenue, Edmonton, Alberta T6G 2J3. Telephone 780-407-1680, fax 780-407-7136, e-mail sarahforgie@cha.ab.ca

Received and accepted for publication August 28, 2007
} 


\section{DIAGNOSIS}

The patient was initially started on piperacillin/tazobactam and vancomycin, while awaiting culture results. The single, initial blood culture drawn from the central venous catheter grew Gram-positive bacilli with spores in slightly over $10 \mathrm{~h}$. On subculture, the organism grew as large, rough beta-hemolytic colonies on sheep blood agar. The bacteria were motile and lecithinase-positive, and failed to grow on MacConkey agar. Based on these microbiological laboratory findings, the organism was identified as Bacillus cereus. The organism was susceptible to vancomycin, and the piperacillin/tazobactam was discontinued. The blood cultures were negative by the third day of treatment, and the vancomycin was continued for 10 days. His central line was removed five days after admission and he remained well throughout his stay in hospital.

\section{DISCUSSION}

Members of the genus Bacillus are aerobic, Gram-positive, spore-forming rods. Bacillus spores are commonly found in both nature (water, dust and soil) and in the hospital setting (1). Despite their ubiquity, most Bacillus species do not cause significant infections and are often considered to be a contaminant when recovered from clinical specimens. However, in certain populations, such as immunosuppressed individuals, neonates, injection drug users or patients with prosthetic material such as intraventricular shunts, these organisms should not be dismissed as contaminants when isolated from certain clinical sites $(1,2)$.

$B$ cereus is best known for causing enterotoxin-related food poisoning syndromes. B cereus is widespread in the food industry, and transmission occurs from contaminated foods and improper handling of foods. Fried rice is a frequent source of B cereus food poisoning; symptoms develop from intoxication and not from ingestion of $B$ cereus vegetative cells. Because our patient frequently ate fried rice, it is possible that ingestion of $B$ cereus vegetative cells, and subsequent translocation, may have been the source of his bacteremia. There has been a positive correlation found between tea consumption and $B$ cereus bacteremia in children with neutropenia (tea leaves are often contaminated with $B$ cereus) (3). Additionally, many patients with $B$ cereus bacteremia have gastrointestinal signs or symptoms at the time of presentation, and a subset have positive stool or rectal swabs for the organism (2).

Another source of this patient's bacteremia may have been extraluminal colonization of the catheter, because $B$ cereus has been linked to skin infections (1). Although superficial swabs from the site did not show Gram-positive bacilli on Gram stain or culture, the organism may have gained access through contaminated gauze or linens (4). The child's line insertion site was routinely cleansed with alcohol swabs, but alcohol will not eradicate $B$ cereus spores. Furthermore, the alcohol itself may have been contaminated; two case reports have shown $B$ cereus contamination of alcohol cotton swabs and $70 \%$ ethyl alcohol solution $(5,6)$. B cereus has also been found in swimming pools and hot springs $(7,8)$. Because the child had been swimming for the first time two weeks before his bacteremia, the pool water may have been the source of extraluminal colonization.

Finally, intraluminal colonization of his central line may have been the source of the bacteremia. His central line was removed because it is recommended to do so in the setting of B cereus bacteremia (2). However, it is debatable whether it was necessary, because he cleared his bacteremia with medical therapy alone, and he was clinically stable (9).

With respect to treatment, $B$ cereus produces beta-lactamases and is, therefore, resistant to beta-lactam antimicrobial agents (1). B cereus is generally susceptible to aminoglycosides, clindamycin, vancomycin, chloramphenicol and erythromycin (1). In this particular case, the cultured B cereus was susceptible to clindamycin, gentamicin and vancomycin. The patient was kept on a 10-day course of vancomycin, which was effective in eradicating the bacteremia.

\section{CONCLUSION}

It is important for clinicians to be familiar with $B$ cereus as a cause of bacteremia in the immunocompromised patient, because there can be serious consequences such as sepsis or endocarditis. Risk factors for development of B cereus bacteremia may involve translocation of the organism from the gut or invasion from contaminated skin in the setting of immunosuppression, prematurity or indwelling prosthetic material. Treatment with vancomycin is usually effective, and for more severe infections, a carbapenem or an aminoglycoside may be added (1). If a central line is present, removal is recommended for persistent bacteremias (2).

\section{REFERENCES}

1. Drobniewski FA. Bacillus cereus and related species. Clin Microbiol Rev 1993;6:324-38.

2. Gaur AH, Patrick CC, McCullers JA, et al. Bacillus cereus bacteremia and meningitis in immunocompromised children. Clin Infect Dis 2001;32:1456-62.

3. El Saleeby CM, Howard SC, Hayden RT, McCullers JA. Association between tea ingestion and invasive Bacillus cereus infection among children with cancer. Clin Infect Dis 2004;39:1536-9.

4. Barrie D, Wilson JA, Hoffman PN, Kramer JM. Bacillus cereus meningitis in two neurosurgical patients: An investigation into the source of the organism. J Infect 1992;25:291-7.

5. Hsueh PR, Teng LJ, Yang PC, Pan HL, Ho SW, Luh KT. Nosocomial pseudoepidemic caused by Bacillus cereus traced to contaminated ethyl alcohol from a liquor factory. J Clin Microbiol 1999;37:2280-4.

6. Berger SA. Pseudobacteremia due to contaminated alcohol swabs. J Clin Microbiol 1983;18:974-5

7. Itah AY, Ekpombok MU. Pollution status of swimming pools in south-south zone of southeastern Nigeria using microbiological and physiochemical indices. Southeast Asian J Trop Med Public Health 2004;35:488-93

8. Schiemann DA. Experiences with bacteriological monitoring of pool water. Infect Control 1985;6:413-7.

9. Lane RK, Matthay AM. Central line infections. Curr Opin Crit Care 2002;8:441-8. 


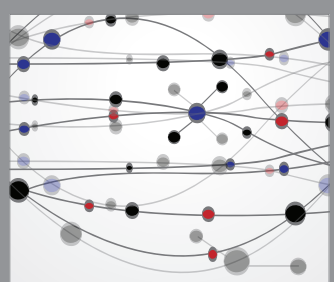

The Scientific World Journal
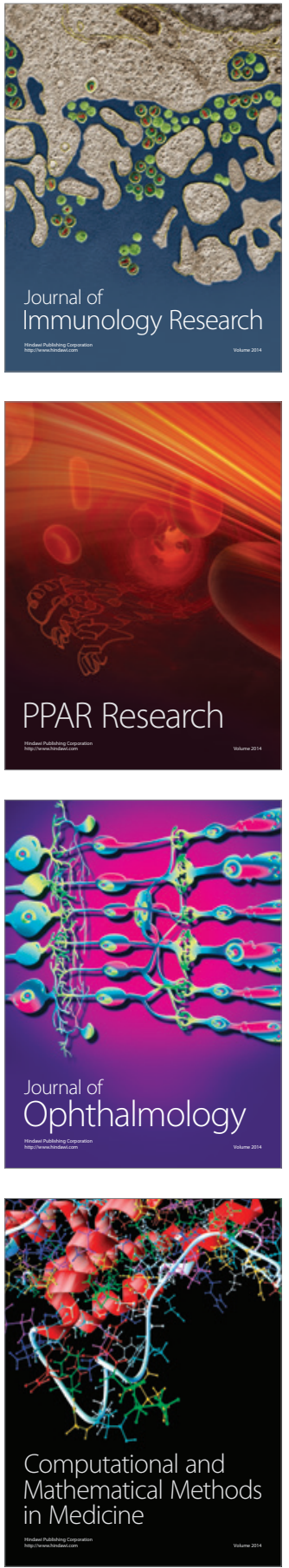

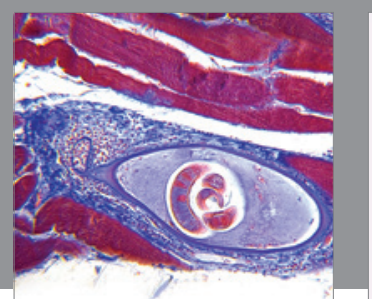

Gastroenterology Research and Practice

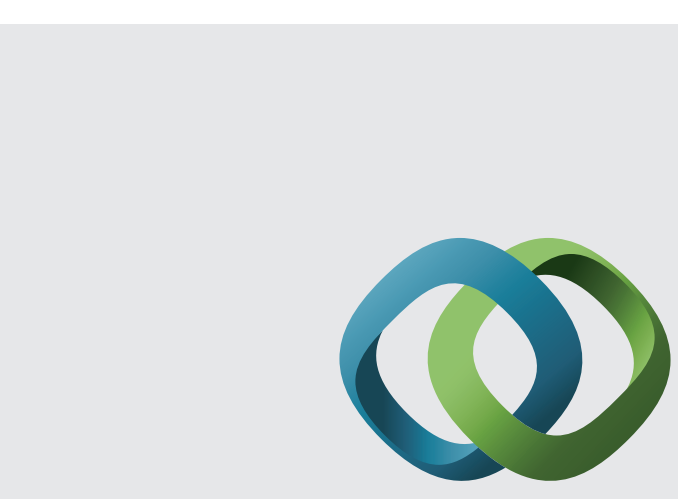

\section{Hindawi}

Submit your manuscripts at

http://www.hindawi.com
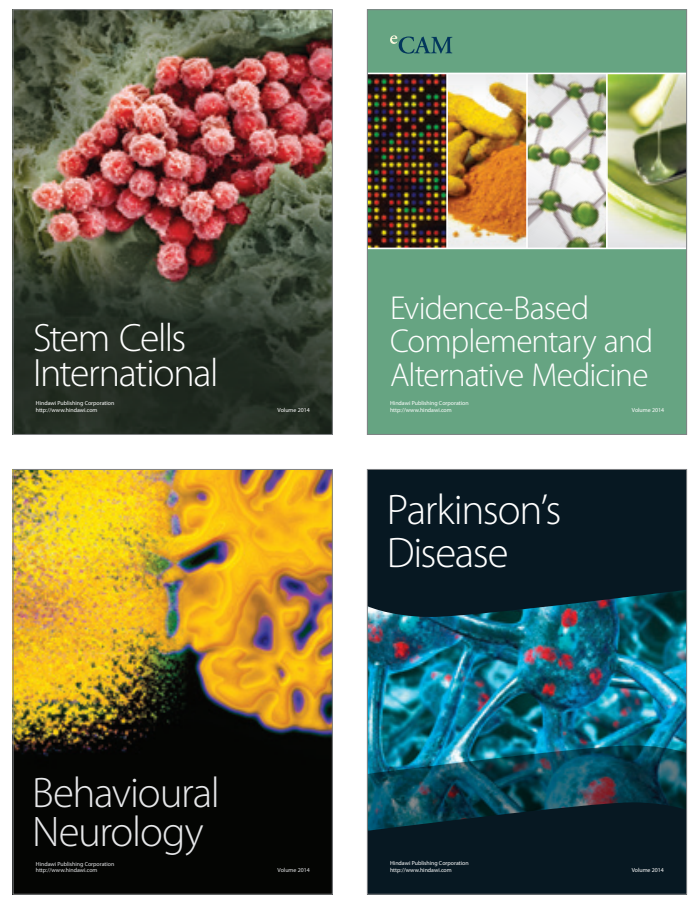
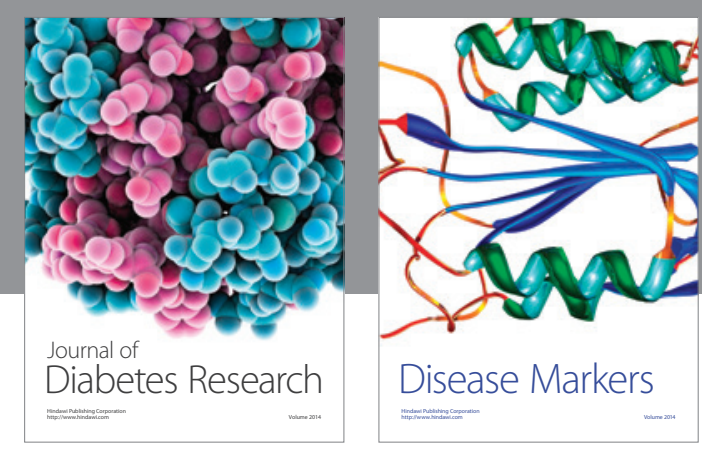

Disease Markers
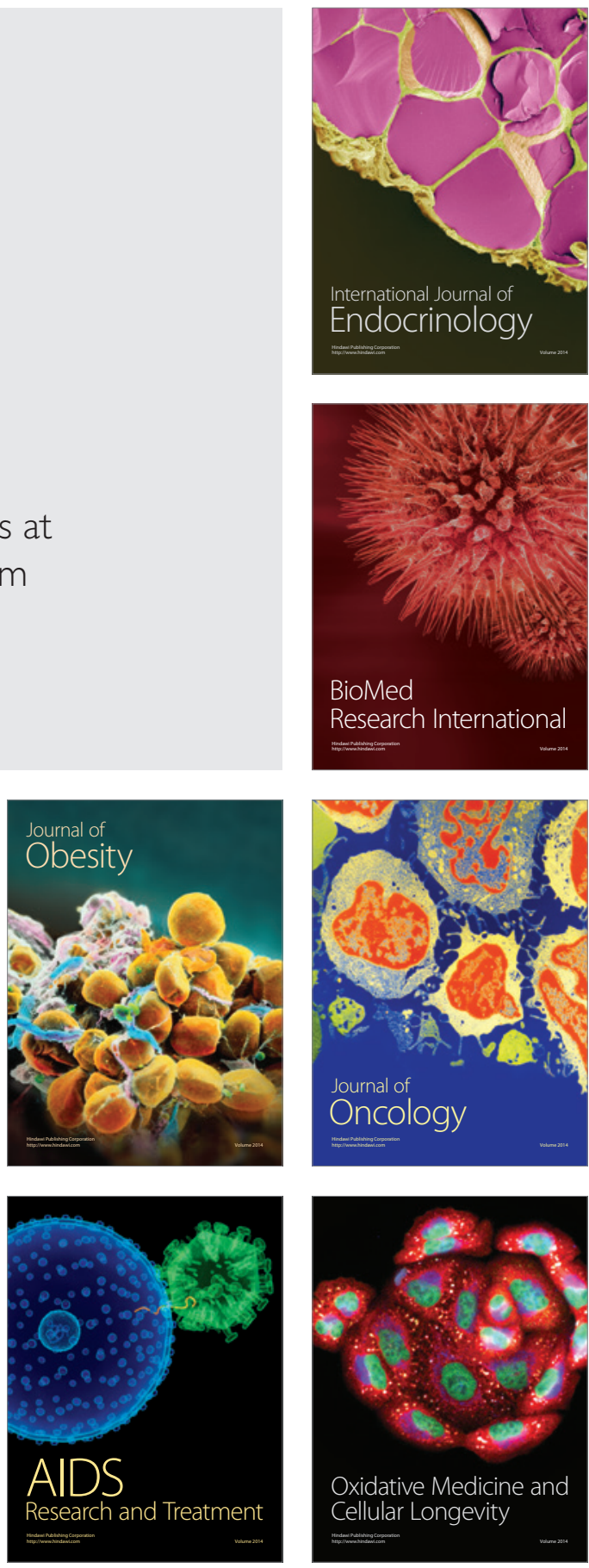